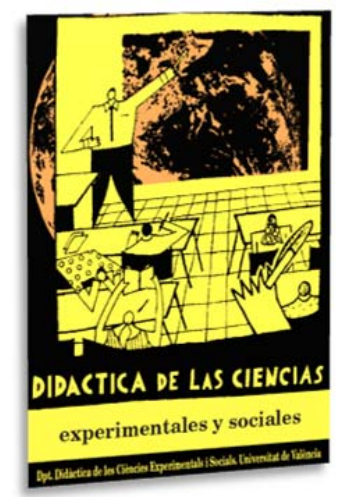

\title{
Smartphones y caída libre: Diseño y evaluación de una experiencia práctica
}

\author{
Smartphones and free fall: Design and \\ evaluation of a practical experience
}

DOI: $10.7203 / D C E S .37 .15441$

\author{
Anna R. Esteve \\ Universitat de València, anna.esteve@uv.es \\ ORCID iD: https://orcid.org/0000-0003-0309-5079 \\ Aina Benavent \\ Universitat de València, aibegi@alumni.uv.es \\ Jordi Solbes \\ Universitat de València, jordi.solbes@uv.es \\ ORCID iD: https://orcid.org/0000-0002-8220-209X
}

\begin{abstract}
RESUMEN: Este trabajo presenta el diseño y evaluación de una experiencia práctica sobre el movimiento de caída libre basada en la utilización de smartphones para comprobar si el aprendizaje de los estudiantes sobre este movimiento mejora al utilizar este tipo de dispositivos móviles. La evaluación de la experiencia práctica se ha realizado mediante un diseño pre-post en el que 47 estudiantes, matriculados en la asignatura "Física y Química” de $4^{\circ}$ de ESO, han contestado a un cuestionario con 6 preguntas de respuesta libre. Los resultados muestran que la experiencia práctica propuesta es eficaz ya que se obtiene una mejora estadísticamente significativa entre el pre-test y el post-test, tanto a nivel global como en cada una de las preguntas del cuestionario.
\end{abstract}

PAlabras ClAve: smartphones, caída libre, experiencia práctica, investigación en el aula, diseño prepost.

ABSTRACT: This work presents the design and evaluation of a practical experience on free fall based on the use of smartphones to check if the students' learning about this motion improves when using these devices. The evaluation of the practical experience has been done with a pre-post design in which 47 students, enrolled in the "Physics and Chemistry" course of the 4th year of compulsory high school (ESO, by its Spanish acronym), have filled in a questionnaire with 6 free answer questions. The results show that the proposed practical experience is effective since a statistically significant improvement is obtained between the pre-test and the post-test, both globally and in each of the questions of the questionnaire.

KEYWORDS: smartphones, free fall, practical experience, classroom research, pre-post design.

Fecha de recepción: junio de 2019

Fecha de aceptación: julio de 2019

Este trabajo ha sido financiado por el Ministerio de Ciencia, Innovación y Universidades y el Fondo Europeo de Desarrollo Regional (FEDER) a través del proyecto EDU2015-69701-P. Los autores desean agradecer al alumnado y al profesorado de los institutos de educación secundaria involucrados en este estudio su participación en el mismo. 


\section{INTRODUCCIÓN}

La alfabetización científica, junto con el lenguaje y la alfabetización artística, son la base para la formación de ciudadanos responsables y el desarrollo socio-económico de cualquier país en un mundo cada vez más globalizado (Hazelkorn et al., 2015). Sin embargo, se ha observado en los últimos años una falta de interés del alumnado hacia las asignaturas de Ciencias y Tecnología, y muy especialmente hacia la Física y las Matemáticas (OECD, 2006; Solbes, Montserrat y Furió, 2007), lo que puede suponer, a largo plazo, una menor capacidad para innovar e investigar de muchos países europeos (Rocard et al., 2007).

Diversos autores han puesto de manifiesto que, aunque las actitudes hacia la ciencia son favorables durante la infancia, el desinterés del alumnado aumenta con la edad, lo que provoca un abandono de los estudios científico-técnicos en los cursos superiores de la educación secundaria, especialmente en física, química o matemáticas (Simpson y Oliver, 1990; Pitburn y Baker, 1993; Osborne, Driver y Simon, 1998; Pell y Jarvis, 2001; Murphy y Beggs, 2003; George, 2006; Robles, Solbes, Cantó y Lozano, 2015). Además, este desinterés tiene múltiples causas, como la forma en la que se enseñan las ciencias, la imagen negativa que tiene el alumnado sobre las ciencias (al pensar que es aburrida, difícil, que no tiene utilidad o que es causa de los problemas medioambientales) o por los estereotipos de género (Solbes, Montserrat y Furió, 2007; Osborne y Dillon, 2008; Vázquez y Manassero, 2008).

Por otra parte, la enseñanza de la cinemática plantea dificultades a los estudiantes debido principalmente a las concepciones alternativas que posee el alumnado y que entran en conflicto con los contenidos escolares conceptuales, así como a la complejidad formal que normalmente es utilizada en el estudio de la cinemática (Champagne, Klopfer y Anderson, 1980). Estas dificultades pueden estar presentes desde el inicio de su estudio hasta incluso el inicio de estudios universitarios de ciencias e ingenierías (Furió, Solbes y Carrascosa, 2006; Jiménez y Guirao, 2007; Fuentes, 2016). Entre las dificultades relacionadas con la cinemática que permite abordar este trabajo podemos mencionar: pensar que un objeto caerá más rápido cuanto más pese (al considerar la fuerza como causa del movimiento), confundir velocidad y aceleración, confundir los signos de la aceleración (considerándola positiva cuando el objeto baja y negativa cuando sube), etc. (Viennot, 1979; Sebastià, 1984; Halloun y Hestenes, 1985; Carrascosa y Gil, 1992).

No obstante, determinados métodos de enseñanza (por ejemplo, métodos que permitan a los estudiantes establecer vínculos entre lo aprendido en la escuela y su vida cotidiana, métodos basados en la indagación por parte de los estudiantes, etc.) pueden ayudar a aumentar la motivación y el interés del alumnado por el estudio de las ciencias (Hasni y Potvin, 2015), así como a contribuir a superar las dificultades de la enseñanza de la cinemática. Así, se pueden encontrar propuestas de este tipo para la enseñanza de la Física en la educación secundaria que incluyen la incorporación de temas actuales, la historia de la Física y las relaciones Ciencia-Tecnología-Sociedad (CTS) o la realización de experimentos y experiencias prácticas (Gil y Solbes, 1993; Ostermann y Moreira, 2000; Caamaño, 2003; Solbes y Vilches, 2004; Lozano y Solbes, 2014).

Las experiencias prácticas suponen un complemento imprescindible de los contenidos conceptuales, pero las condiciones en los centros de enseñanza no siempre son las idóneas para realizar las tradicionales prácticas de laboratorio, por lo que en los últimos años ha aparecido la alternativa de utilizar smartphones y/o tablets, que suelen estar disponibles fácilmente y que el alumnado relaciona con su vida cotidiana, para realizar experiencias prácticas en las aulas o en los laboratorios. Así, el profesorado de Física y Química puede usar el interés del alumnado en estos dispositivos para proponer experiencias prácticas que utilicen los sensores que smartphones y tablets llevan incorporados (acelerómetro, giroscopio, sensor de luz, etc.) como herramientas de medida tanto en el laboratorio o en el aula como en actividades fuera del aula para aumentar la motivación del alumnado (Kuhn y Vogt, 2013a). De esta forma, el alumnado se da cuenta de que lo 
que aprende en las clases de Física lo puede aplicar en su vida cotidiana (Lozano y Solbes, 2014; González et al., 2015), lo que influye positivamente en su motivación (Kuhn y Müller, 2015).

En la bibliografía reciente se pueden encontrar numerosas propuestas de experiencias prácticas de Física basadas en la utilización de smartphones y/o tablets como herramientas de medida en experiencias de mecánica (Kuhn y Vogt, 2012; Vogt y Kuhn, 2012a, 2012b; CastroPalacio, Velázquez-Abad, Giménez y Monsoriu, 2013; Vogt y Kuhn, 2014; Vieyra y Vieyra, 2014; Hochberg, Gröber, Kuhn y Müller, 2014; Monteiro, Stari, Cabeza y Martí, 2015), acústica (Parolin y Pezzi, 2013; Kuhn y Vogt, 2013b; Kuhn, Vogt y Hirth, 2014; Parolin y Pezzi, 2015; González y González, 2016; Di Laccio Caceres et al., 2017) u óptica (Sitar, 2012; Thoms, Colicchia y Girwidz, 2013; Monteiro, Stari, Cabeza y Martí, 2017; Sans et al., 2017; Salinas, Giménez, Monsoriu y Castro-Palacio, 2018). Sin embargo, hasta ahora no se ha empezado a comprobar la eficacia de la utilización de smartphones y/o tablets como herramientas de medida en experiencias prácticas en el aprendizaje de la Física por parte del alumnado.

Por lo tanto, el objetivo de la investigación que se presenta en este trabajo ha sido comprobar si una experiencia práctica sobre el movimiento de caída libre (movimiento de un cuerpo bajo la acción del campo gravitatorio terrestre y en ausencia de la resistencia del aire) basada en la utilización de smartphones puede ayudar al alumnado a superar sus concepciones alternativas sobre la caída libre y comprender mejor los conceptos relacionados con este movimiento. Para ello, se ha realizado una secuencia de actividades con esta experiencia práctica y un diseño pre-post para evaluar en qué medida ayuda a mejorar el aprendizaje del alumnado sobre la caída libre.

\section{Propuesta de enseñanza}

La experiencia práctica sobre el movimiento de caída libre ha sido diseñada a partir de las experiencias propuestas por Vogt y Kuhn (2012a) y Gil y Di Laccio (2017) (Esteve y Petit, 2019). Esta se basa en la utilización del acelerómetro del smartphone y una aplicación informática específica (app) que permita acceder a los datos registrados por este sensor. En este caso, la app utilizada ha sido Physics Toolbox Sensor Suite, desarrollada por la compañía Vieyra Software (https://www.vieyrasoftware.net/). Esta app, disponible de forma gratuita tanto para el sistema operativo Android como para iOS, permite registrar las medidas realizadas con multitud de sensores (dependiendo de los sensores disponibles en cada dispositivo) y exportar los datos en formato csv para su posterior análisis en una hoja de cálculo. En la experiencia práctica propuesta solo se necesitarán las funciones de "Fuerza G” y “Acelerómetro lineal” de la app.

La propuesta consta de cinco actividades que se desarrollan en 2 sesiones de 1 hora cada una. Durante la primera sesión, se revisan los conceptos básicos relacionados con este movimiento (ecuaciones y gráficas posición-tiempo y velocidad-tiempo del movimiento rectilíneo uniformemente acelerado aplicadas a la caída libre, aceleración de la gravedad y ausencia de la resistencia del aire), el alumnado formula sus hipótesis sobre cómo se mueven los objetos en caída libre y realiza las medidas con los smartphones. Durante la segunda sesión, el alumnado analiza sus medidas con la ayuda de un ordenador y se realiza una discusión grupal sobre los resultados obtenidos.

Las actividades se describen a continuación:

\section{Actividad 1. Formulación de hipótesis sobre la caída libre}

Para introducir la experiencia práctica sobre caída libre se presenta al alumnado una actividad lúdica de la vida real como es una persona haciendo puenting y se plantean las preguntas siguientes:

- La aceleración en la caída libre ¿es constante o cambia?

- ¿Se trata de un movimiento rectilíneo uniformemente acelerado o no?

- ¿Cómo influye la masa del objeto en la velocidad de caída? 
Las hipótesis sobre la caída libre que formule el alumnado a partir de estas preguntas guiarán la investigación durante la experiencia práctica. Aquí se espera que aparezcan como hipótesis de trabajo algunas de las concepciones alternativas sobre este tipo de movimiento mencionadas anteriormente: considerar que la aceleración en la caída libre aumenta (al confundir velocidad y aceleración), pensar que un objeto cae más rápido cuanto más pesa (al considerar la fuerza como causa del movimiento), etc.

A continuación, se revisan los conceptos básicos relacionados con la caída libre (ecuaciones y gráficas posición-tiempo y velocidad-tiempo del movimiento rectilíneo uniformemente acelerado aplicadas a la caída libre, aceleración de la gravedad y ausencia de la resistencia del aire).

\section{Actividad 2. Aprendiendo a usar el acelerómetro del smartphone}

Esta experiencia práctica se basa en la utilización del acelerómetro del smartphone y la app Physics Toolbox Sensor Suite para registrar los datos de este sensor.

Una vez explicada la utilización de la app, el alumnado debe comprobar cómo están orientados los ejes $\mathrm{X}, \mathrm{Y}, \mathrm{Z}$ de su smartphone antes de empezar a utilizar el acelerómetro (Countryman, 2014), ya que esto determinará la orientación en que se deberá dejar caer el dispositivo durante la caída libre. Para ello, se pide al alumnado que observe y anote qué eje marca un valor de $1 \mathrm{G}$ cuando los otros dos ejes marcan un valor de $0 \mathrm{G}$ en la función "Fuerza G" de la app al colocar el smartphone en diferentes posiciones (por ejemplo, apoyado sobre cada uno de los lados del dispositivo). Esta función de la app proporciona una medida intuitiva de la aceleración que produciría la gravedad en un objeto en condiciones ideales (sin atmósfera u otro rozamiento), es decir, $1 \mathrm{G}=9.8 \mathrm{~m} / \mathrm{s}^{2}$. La Imagen 1 muestra un ejemplo de los resultados que se obtienen en esta actividad.

IMAGEN 1. Orientación de los ejes X, Y, Z de un smartphone.

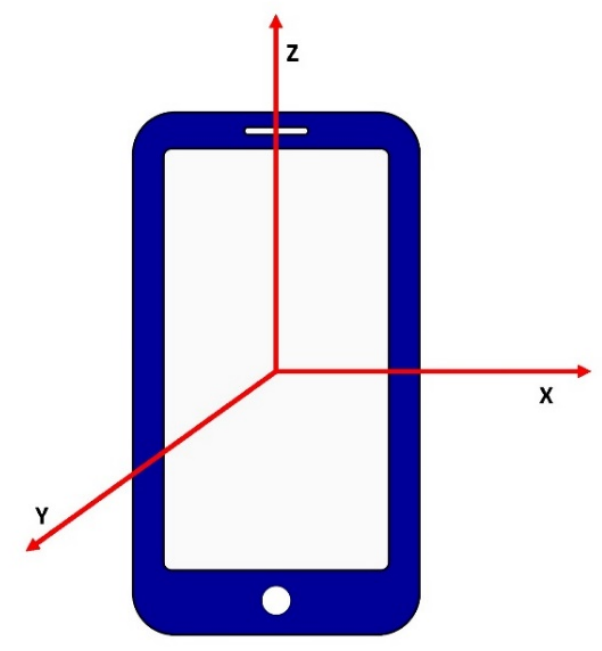

Fuente: Esta imagen es una obra derivada de “Smartphone icon.svg” por G. Hagedorn utilizada bajo CC BY-SA 3.0.

\section{Actividad 3. Análisis de la aceleración de la caída libre}

Una vez el alumnado se ha familiarizado con el uso de la app y del acelerómetro del smartphone, se activa la función “Acelerómetro lineal” y el registro de datos; y se deja caer el dispositivo desde una altura aproximada de 1.5 metros sobre algún elemento protector (cojines, colchonetas o similares) para evitar dañarlo.

Los datos registrados durante la caída libre del smartphone son exportados y analizados en una hoja de cálculo, obteniéndose una gráfica como la que se observa en la Imagen 2. Al principio, el smartphone está en reposo y el acelerómetro detecta la gravedad terrestre $\left(\sim 9.8 \mathrm{~m} / \mathrm{s}^{2}\right)$ ya que está siendo sujetado por el alumnado, un hilo, etc. A continuación, el dispositivo empieza a caer y 
sentirá un efecto equivalente a la ingravidez al estar cayendo con una aceleración igual a la gravedad terrestre, por lo que el acelerómetro no detectará ninguna aceleración. Finalmente, el smartphone aterriza sobre el elemento protector y acaba parándose, por lo que el acelerómetro vuelve a detectar la gravedad terrestre $\left(\sim 9.8 \mathrm{~m} / \mathrm{s}^{2}\right)$ al ser sujetado por el elemento protector.

\section{Actividad 4. Análisis de la influencia de la masa en la caída libre}

La actividad que se plantea a continuación trabaja una de las concepciones alternativas más extendidas sobre la caída libre, la que establece una relación directa entre la masa del cuerpo y su velocidad de caída (Santos y Gras-Martí, 2003).

IMAGEN 2. Aceleración vs. tiempo antes, durante y después de la caída libre del smartphone.

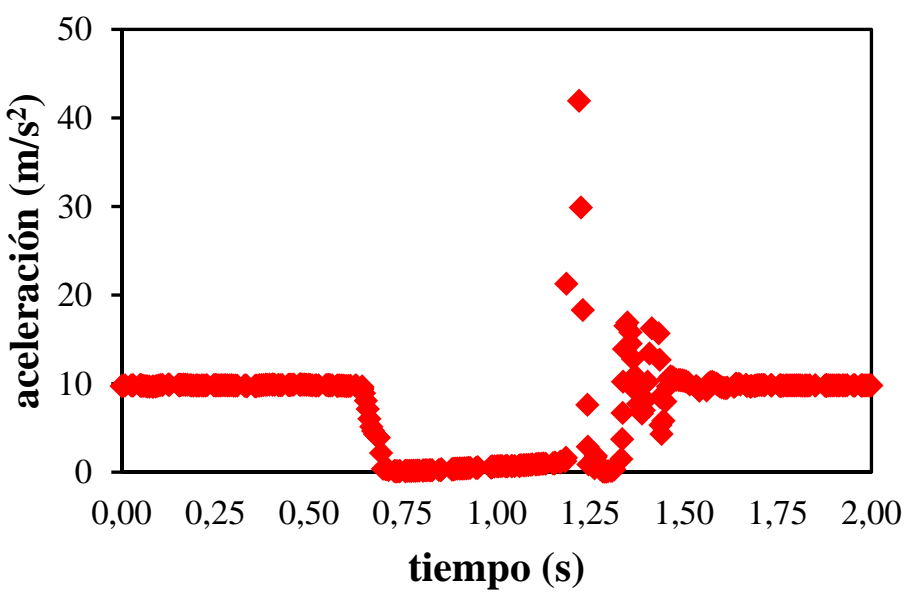

Fuente: elaboración propia.

En primer lugar, se activa la función “Acelerómetro lineal” y el registro de datos de la app y se vuelve a dejar caer el smartphone desde una altura aproximada de 1.5 metros sobre algún elemento protector, midiendo el tiempo que el dispositivo tarda en llegar al suelo con un cronómetro tradicional o con el cronómetro de otro smartphone. Se repite esta medida un total de 3 veces, anotando los valores, así como la masa del dispositivo, en una tabla como la que se puede observar en la Tabla 1. Después, se juntan dos smartphones (por ejemplo, envolviéndolos con un papel o bolsa y cinta adhesiva) y se dejan caer desde una altura aproximada de 1.5 metros sobre algún elemento protector, midiendo el tiempo que tarda el sistema formado por ambos dispositivos en llegar al suelo. Se repite esta medida un total de 3 veces, anotando los valores, así como la masa del sistema formado por ambos dispositivos, en una tabla como la Tabla 1.

TABLA 1. Tabla para el registro de medidas para el análisis de la influencia de la masa en la caída libre.

\begin{tabular}{|l|c|c|c|c|c|c|c|}
\hline \multicolumn{3}{|c|}{ 1 smartphone } & \multicolumn{4}{|c|}{ 2 smartphones } \\
\hline & tiempo (s) & valor medio & masa (g) & & tiempo (s) & valor medio & masa (g) \\
\hline 1 & & & & 1 & & & \\
\cline { 1 - 3 } 2 & & & & 2 & & & \\
\cline { 1 - 3 } 3 & & & 3 & & & \\
\end{tabular}

Fuente: elaboración propia.

A partir de las medidas realizadas, se calcula el tiempo medio de caída para cada uno de los sistemas: 1 smartphone (con masa m) y 2 smartphones (con masa $2 \mathrm{~m}$ aproximadamente). Del análisis de estos resultados se deduce que el tiempo que tarda en llegar al suelo un objeto en caída libre es independiente de la masa del mismo. 
Durante la caída libre (y en ausencia de la resistencia del aire), la aceleración que adquiere un cuerpo se debe exclusivamente a la gravedad, que tiene un valor constante de aproximadamente 9.8 $\mathrm{m} / \mathrm{s} 2$. Así, de acuerdo con las ecuaciones del movimiento rectilíneo uniformemente acelerado, la velocidad de un cuerpo en caída libre viene dada por la expresión $\mathrm{v}=-\mathrm{g} \cdot \mathrm{t}$, donde se observa que esta es independiente de la masa del objeto, por lo que 2 objetos con diferente masa tardarán el mismo tiempo en llegar al suelo al caer desde la misma altura.

\section{Actividad 5. Discusión de los resultados}

Por último, y para concluir la experiencia práctica, se realiza una discusión grupal sobre los resultados obtenidos en las actividades anteriores y se pide al alumnado que conteste las siguientes preguntas:

- ¿Cómo es la aceleración en el movimiento de caída libre? ¿Qué valor tiene?

- ¿Cómo varía la velocidad del smartphone a medida que este se aproxima al suelo?

- ¿Hay diferencia entre el tiempo que tarda en llegar al suelo un smartphone y el tiempo que tardan 2 smartphones unidos? Por lo tanto, ¿cómo influye la masa del objeto en su velocidad de caída?

- ¿Qué aplicaciones prácticas puede tener lo que has aprendido?

En esta discusión se espera que el alumnado reconozca la caída libre como un caso especial de movimiento rectilíneo uniformemente acelerado, donde la aceleración constante con la que caen los objetos es la gravedad terrestre $\left(\sim 9.8 \mathrm{~m} / \mathrm{s}^{2}\right)$, que hace que la velocidad de los objetos aumente a medida que se aproximan al suelo. También se espera que el alumnado ya no piense que los objetos caerán más rápido cuanto más pesen. Además, el alumnado debe ser capaz de proponer aplicaciones prácticas de la caída libre y los métodos aprendidos en esta experiencia práctica como el cálculo de la profundidad del agua en un pozo; el tiempo que se tarda en caer, la velocidad con la que se cae o la longitud de la cuerda haciendo puenting; la altura de un edificio, etc.

\section{Metodología}

Con el fin de comprobar hasta qué punto la experiencia práctica propuesta en el apartado anterior ayuda a mejorar el aprendizaje del alumnado sobre la caída libre, se realizó una evaluación de la misma mediante un diseño pre-post. Para ello, se elaboró un cuestionario a partir de la modificación del Force Concept Inventory (Hestenes, Wells y Swackhamer, 1992) propuesta y validada por Garduño Calderón, López Ortega y Mora (2013). El cuestionario utilizado, cuyas preguntas se muestran en la Tabla 2, tiene seis preguntas de respuesta libre: dos preguntas sobre concepciones alternativas ( 1 y 2), dos preguntas sobre conceptos (3 y 4), una pregunta sobre cómo aplicar los métodos aprendidos en esta experiencia (5) y una pregunta sobre las aplicaciones de la caída libre en la vida cotidiana que conoce el alumnado (6). Las preguntas 1 a 4 son adaptaciones de las propuestas por Garduño Calderón, López Ortega y Mora (2013). Las respuestas del alumnado se clasificaron en categorías distintas de acuerdo con los criterios de análisis que también se muestran en la Tabla 2: las preguntas 1 y 2 en categorías de correctas e incorrectas y las preguntas 3, 4, 5 y 6 en categorías de correctas, parcialmente correctas e incorrectas. Además, la fiabilidad del cuestionario se estimó calculando el alfa de Cronbach, obteniéndose un valor de 0.66, que está muy próximo al valor aceptado generalmente de 0.70 para indicar la consistencia interna del cuestionario.

Un total de 47 estudiantes de la asignatura "Física y Química” de $4^{\circ}$ de ESO (15-16 años) realizaron la experiencia práctica y contestaron al pre-test y al post-test. 
TABLA 2. Preguntas y criterios de análisis del cuestionario pre-post

1. Desde el segundo piso del instituto (8 metros de altura), dejamos caer a la vez dos pelotas de diferente masa. La pelota $\mathrm{X}$ tiene una masa de 100 gramos y la pelota $\mathrm{Y}$ de 50 gramos. Cuando la pelota Y haya descendido 2 metros, ¿dónde estará la pelota X?

CORRECTA $\quad$ La pelota X habrá descendido también $2 \mathrm{~m}$.

INCORRECTA

La pelota $\mathrm{X}$ estará más abajo que la pelota $\mathrm{Y}$ porque tiene mayor masa.

2. Desde el segundo piso del instituto (8 metros de altura), dejamos caer a la vez dos pelotas de diferente masa: una pelota $\mathrm{X}$ de 100 gramos y otra pelota $\mathrm{Y}$ de 50 gramos. ¿Qué pelota llegará antes al suelo?

\begin{tabular}{c|c} 
CORRECTA & Las dos pelotas caerán a la vez. \\
\hline INCORRECTA & La pelota X llegará antes al suelo porque tiene mayor masa.
\end{tabular}

3. Desde el segundo piso del instituto (8 metros de altura), dejamos caer a la vez dos pelotas de diferente masa: una pelota $\mathrm{X}$ de 100 gramos y otra pelota $\mathrm{Y}$ de 50 gramos. ¿Cómo variará su velocidad a lo largo de la caída de 8 metros? ¿Qué pelota alcanzará más velocidad?

\begin{tabular}{|c|c|}
\hline CORRECTA & $\begin{array}{c}\text { La respuesta afirma que la velocidad de un cuerpo aumenta a medida que el } \\
\text { tiempo de caída también aumenta y que las dos pelotas alcanzarán la misma } \\
\text { velocidad. }\end{array}$ \\
\hline $\begin{array}{c}\text { PARCIALMENTE } \\
\text { CORRECTA }\end{array}$ & $\begin{array}{c}\text { La respuesta afirma que la velocidad va aumentando o que las dos pelotas } \\
\text { alcanzarán la misma velocidad. }\end{array}$ \\
\hline INCORRECTA & La respuesta no responde correctamente ninguna de las dos preguntas \\
planteadas.
\end{tabular}

4. Si dejamos caer a la vez dos monedas, una moneda $\mathrm{X}$ desde el segundo piso del instituto y otra moneda Y desde el primer piso, ¿cómo será la aceleración de ambas monedas al final de la caída? ¿Qué aceleración tendrán?

\begin{tabular}{|c|c|}
\hline CORRECTA & $\begin{array}{c}\text { La aceleración que actúa es constante y se corresponde con la aceleración de la } \\
\text { gravedad }\left(9.8 \mathrm{~m} / \mathrm{s}^{2}\right) .\end{array}$ \\
\hline $\begin{array}{c}\text { PARCIALMENTE } \\
\text { CORRECTA }\end{array}$ & $\begin{array}{c}\text { La respuesta afirma que la aceleración es constante, pero no la relaciona con la } \\
\text { aceleración de la gravedad. }\end{array}$ \\
\hline INCORRECTA & La respuesta afirma que la aceleración en la caída libre varía. \\
\hline
\end{tabular}

5. A la hora del patio, un compañero te deja caer la manzana del almuerzo desde la ventana de clase. El profesor de guardia de patio os ve y os dice que, si no queréis estar a la hora del patio dentro del aula, tenéis que medir la velocidad con la que ha caído la manzana. ¿Cómo lo haríais?

\begin{tabular}{|c|c|}
\hline CORRECTA & $\begin{array}{c}\text { La respuesta afirma que estimando la altura y el tiempo que tarda en caer el } \\
\text { bocadillo, y dado que la aceleración es constante y es la de la gravedad (9.8 } \\
\mathrm{m} / \mathrm{s}^{2} \text { ), con las ecuaciones de la caída libre se puede calcular. }\end{array}$ \\
\hline $\begin{array}{c}\text { PARCIALMENTE } \\
\text { CORRECTA }\end{array}$ & $\begin{array}{r}\text { La respuesta indica que se deben utilizar las ecuaciones de la caída libre, pero } \\
\text { no se explica el procedimiento correctamente. }\end{array}$ \\
\hline INCORRECTA & $\begin{array}{c}\text { La respuesta no hace un razonamiento lógico o afirma que estimando la altura } \\
\text { y el tiempo que tarda en caer el bocadillo se calcula la velocidad de caída con } \\
\text { la ecuación } \mathrm{v}=\mathrm{d} / \mathrm{t} .\end{array}$ \\
\hline
\end{tabular}

6. ¿Qué aplicaciones prácticas piensas que puede tener lo que vas a aprender sobre el movimiento de caída libre?

\begin{tabular}{|c|c|}
\hline CORRECTA & $\begin{array}{c}\text { La respuesta proporciona dos o más aplicaciones prácticas (p.ej., calcular la } \\
\text { profundidad del agua en un pozo; el tiempo que se tarda en caer o la velocidad } \\
\text { con la que se cae haciendo puenting; la altura de un edificio, etc). }\end{array}$ \\
\hline $\begin{array}{c}\text { PARCIALMENTE } \\
\text { CORRECTA }\end{array}$ & La respuesta proporciona solo una aplicación práctica. \\
\hline INCORRECTA & La respuesta no proporciona ninguna aplicación práctica. \\
\hline
\end{tabular}


El cuestionario fue respondido antes de realizar la experiencia práctica (pre-test) y después, tras pasar 15 días desde su realización (post-test). De esta forma, se pretende comparar los resultados obtenidos en el post-test con los del pre-test, tanto de forma global como pregunta a pregunta, para ver si hay diferencias significativas entre ambos. Para el tratamiento estadístico del cuestionario, realizado con el programa SPSS 24 y con R 1.1.419 (Lukke Sweet, 2017; R Core Team, 2017), las respuestas correctas se puntuaron como 2, las parcialmente correctas como 1 y las incorrectas como 0.

\section{RESULTADOS Y DISCUSIÓN}

El cuestionario utilizado en el diseño pre-post tiene 2 preguntas con respuestas correctas (2) e incorrectas (0) y 4 preguntas con respuestas correctas (2), parcialmente correctas (1) e incorrectas (0), por lo que la puntuación global máxima es 12 y la mínima, 0. En este caso, tal y como se puede observar en la Tabla 3, la media global obtenida por el alumnado en el pre-test es 3.21 (con una desviación estándar de 2.64) y en el post-test, 8.43 (con una desviación estándar de 2.10), por lo que se observa un aumento considerable en la puntuación global obtenida por el alumnado en el cuestionario después de realizar la experiencia práctica basada en la utilización de smartphones. Sin embargo, se deben realizar pruebas estadísticas para comprobar si estas diferencias entre las medias globales del pre-test y el post-test son estadísticamente significativas.

Como se trata de muestras relacionadas (diseño pre-post) que no siguen una distribución normal (según la prueba de Shapiro-Wilk, con $\mathrm{p}<0.05$ ), se realiza la prueba no paramétrica de rangos con signo de Wilcoxon, cuyo resultado es: $Z=-5.821, p=0.000$. Como $p<0.05$, se puede afirmar que existen diferencias estadísticamente significativas entre las medias globales del pre-test y del post-test, por lo que se ha producido una mejora significativa al realizar la experiencia práctica basada en la utilización de smartphones. Además, al calcular el tamaño del efecto r (Rosenthal, 1994) se obtiene un valor de 0.6, es decir, un efecto grande.

TABLA 3. Media global (y desviación estándar), significación estadística y tamaño del efecto del cuestionario pre-post.

\begin{tabular}{|c|c|c|c|c|}
\hline & $\begin{array}{c}\text { Media } \\
(\text { desv. estándar })\end{array}$ & $\begin{array}{c}\text { Diferencia estadísticamente significativa } \\
(\mathrm{p}<0.05)\end{array}$ & \multicolumn{2}{|c|}{ Tamaño del efecto } \\
\hline pre-test & $3.21(2.64)$ & sí & 0.6 & grande \\
\hline post-test & $8.43(2.10)$ & & sín
\end{tabular}

Fuente: elaboración propia.

En la imagen 3 se muestran los porcentajes de respuestas correctas, parcialmente correctas e incorrectas del alumnado en el pre-test y el post-test. Para las preguntas sobre concepciones alternativas de la caída libre $\left(\begin{array}{lll}1 & \text { y } & 2\end{array}\right)$, el porcentaje de respuestas incorrectas disminuye considerablemente en el post-test (del 55.3\% y 59.6\%, respectivamente, al 8.5\%), aumentando así el porcentaje de respuestas correctas hasta el 91.5\%. Para las preguntas sobre conceptos de este tipo de movimiento (3 y 4), el porcentaje de respuestas incorrectas también disminuye considerablemente en el post-test (del $42.6 \%$ al $10.6 \%$ y del $76.6 \%$ al $19.1 \%$, respectivamente), aumentando el porcentaje de respuestas correctas (hasta el $51.1 \%$ y el $57.4 \%$, respectivamente) y manteniéndose casi invariable el porcentaje de respuestas parcialmente correctas (alrededor del $40 \%$ y del 20\%, respectivamente). Para las preguntas sobre cómo aplicar los métodos aprendidos en la experiencia práctica (5) o las aplicaciones en la vida cotidiana de la caída libre (6), la disminución del porcentaje de respuestas incorrectas en el post-test es de nuevo considerable (del $89.4 \%$ al 51.1\% y del $59.6 \%$ al $17 \%$, respectivamente), aumentando principalmente el porcentaje de respuestas 
correctas (hasta el $27.7 \%$ y el $38.3 \%$, respectivamente) y con una variación inferior al $11 \%$ en el porcentaje de respuestas parcialmente correctas.

IMAGEN 3. Porcentaje de respuestas correctas, parcialmente correctas e incorrectas de cada pregunta del cuestionario pre-post.

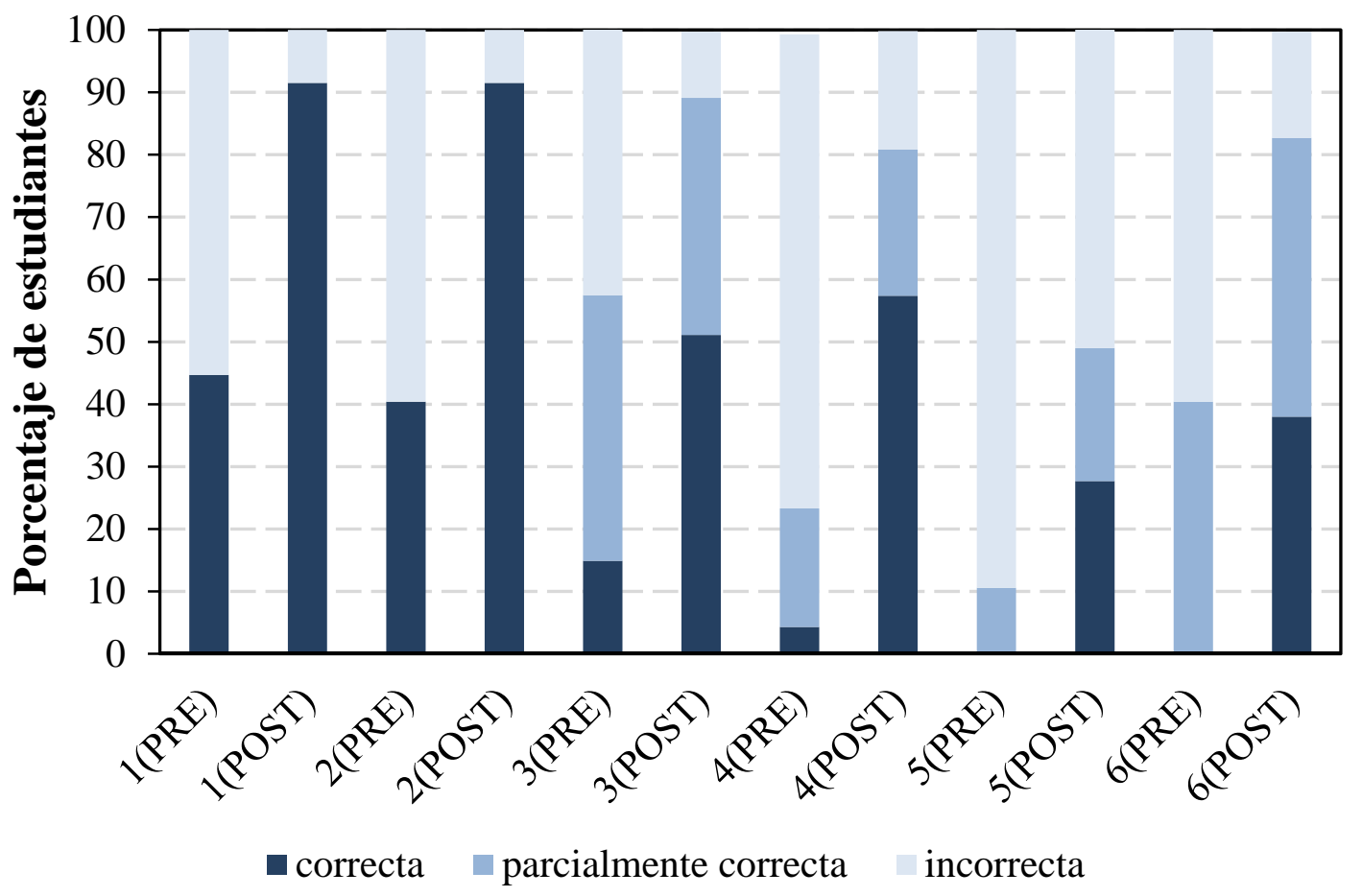

Fuente: elaboración propia.

Para comprobar si las diferencias observadas entre el pre-test y el post-test para cada una de las preguntas del cuestionario son estadísticamente significativas, no se puede aplicar la misma prueba estadística a todas ellas ya que tienen distinto número de categorías. Para las preguntas con dos categorías ( 1 y 2), se utiliza la prueba no paramétrica de McNemar y para las preguntas con tres categorías (3, 4, 5 y 6), se utiliza la prueba no paramétrica de Stuart-Maxwell. La Tabla 4 muestra si hay diferencias significativas entre el pre-test y el post-test, así como el tamaño del efecto, para cada una de las preguntas del cuestionario.

TABLA 4. Significación estadística y tamaño del efecto para cada pregunta del cuestionario pre-post.

\begin{tabular}{|c|c|c|c|c|}
\hline Pregunta & $\mathrm{p}$ & $\begin{array}{c}\text { Diferencia estadísticamente significativa } \\
(\mathrm{p}<0.05)\end{array}$ & \multicolumn{2}{|c|}{ Tamaño del efecto } \\
\hline 1 & 0.000 & sí & 0.46 & moderado \\
\hline 2 & 0.000 & sí & 0.49 & moderado - grande \\
\hline 3 & 0.000 & sí & 0.44 & moderado \\
\hline 4 & 0.000 & sí & 0.52 & grande \\
\hline 5 & 0.000 & sí & 0.41 & moderado \\
\hline 6 & 0.000 & sí & 0.47 & moderado \\
\hline
\end{tabular}

Fuente: elaboración propia.

En la Tabla 4 se observa que existen diferencias estadísticamente significativas entre el pretest y el post-test para cada una de las preguntas del cuestionario, por lo que se ha producido una mejora significativa al realizar la experiencia práctica basada en la utilización de smartphones en todos los aspectos tratados en la misma (concepciones alternativas, conceptos, aplicación de los 
métodos aprendidos y conocimiento de aplicaciones de la caída libre en la vida cotidiana). Además, al calcular el tamaño del efecto $\mathrm{r}$ (Rosenthal, 1994) se obtiene un valor superior a 0.4 en todas las preguntas, es decir, un efecto entre moderado y grande.

\section{Conclusiones}

En los últimos años han aparecido múltiples propuestas para utilizar los omnipresentes smartphones en experiencias prácticas de Física ya que estos dispositivos pueden ayudar a mejorar la motivación y el interés del alumnado por esta asignatura. Por lo que en este trabajo se ha diseñado una experiencia práctica sobre el movimiento de caída libre basada en la utilización de smartphones con el objetivo de ayudar al alumnado a superar sus concepciones alternativas sobre la caída libre y comprender mejor los conceptos relacionados con este movimiento. Las actividades propuestas, que se desarrollan en 2 sesiones de 1 hora cada una, permiten al alumnado formular hipótesis sobre cómo se mueven los objetos en caída libre, utilizar sus smartphones como herramientas de medida mediante una app específica que les permite acceder a los datos registrados por el acelerómetro integrado en sus dispositivos, así como analizar y discutir en grupo los resultados obtenidos.

Se ha realizado también una evaluación de la experiencia práctica propuesta con un cuestionario pre-post que respondieron 47 estudiantes de $4^{\circ}$ de ESO para evaluar en qué medida este tipo de propuestas ayuda a mejorar su aprendizaje sobre la caída libre. Los resultados obtenidos muestran una mejora estadísticamente significativa entre las medias globales del cuestionario prepost, con un tamaño del efecto grande, lo que indica que la experiencia práctica propuesta sobre el movimiento de caída libre basada en la utilización de smartphones consigue su objetivo de ayudar al alumnado en su aprendizaje sobre este tipo de movimiento. Además, también se observan diferencias estadísticamente significativas para cada una de las preguntas del cuestionario pre-post, con un tamaño del efecto entre moderado y grande, por lo que esta propuesta ayuda al alumnado a superar sus concepciones alternativas sobre la influencia de la masa en la caída libre, también a comprender mejor los conceptos relacionados con este movimiento, así como a conocer las aplicaciones de lo aprendido durante la experiencia práctica en la vida cotidiana.

Por lo tanto, en este trabajo se ha comprobado cómo una propuesta de enseñanza de la Física basada en la utilización de smartphones como herramientas de medida en experiencias prácticas puede ayudar a mejorar el aprendizaje del alumnado sobre un tema concreto. Sin embargo, todavía queda mucho trabajo por realizar en esta área ya que, aunque existen numerosas propuestas de experiencias prácticas con smartphones y/o tablets, no se han integrado dentro de propuestas constructivistas de enseñanza-aprendizaje de la Física ni se ha evaluado la eficacia de las mismas con muestras de estudiantes ni en distintos niveles educativos.

\section{Referencias}

Caamaño, A. (2003). Los trabajos prácticos en ciencias. En M.P. Jimenez-Aleixandre (coord.), Enseñar ciencias (pp. 95-118). Barcelona: Graó.

Carrascosa, J. y Gil, D. (1992). Concepciones alternativas en mecánica. Enseñanza de las Ciencias, 10(3), 314-328.

Castro-Palacio, J.C., Velázquez-Abad, L., Giménez, F. y Monsoriu, J.A. (2013). A quantitative analysis of coupled oscillations using mobile accelerometer sensors. European Journal of Physics, 34, 737-744. DOI: 10.1088/0143-0807/34/3/737.

Champagne, A.B., Klopfer, L.E. y Anderson, J.H. (1980). Factors influencing the learning of classical mechanics. American Journal of Physics, 48, 1074-1079. DOI: 10.1119/1.12290. 
Countryman, C.L. (2014). Familiarizing students with the basics of a smartphone's internal sensors. The Physics Teacher, 52, 557-559. DOI: 10.1119/1.4902204.

Di Laccio Caceres, J., Vitale, L.G., Alonso Suárez, R., Pérez, N. y Gil, S. (2017). Estudio del Efecto Doppler usando Smartphones. Revista Eureka Sobre Enseñanza Y Divulgación De Las Ciencias, 14(3), 637-646.

Esteve, A.R. y Petit, M.F. (2019). Experiencias prácticas de Física usando los sensores de un smartphone. En J. Solbes y M.R. Jiménez-Liso (eds.), Propuestas de educación científica basadas en la indagación y modelización en contexto (pp. 147-167). Valencia: Tirant.

Fuentes C. (2016). Preconceptos de cinemática y fuerza en estudiantes que inician sus estudios de ingeniería. Revista Electrónica de Enseñanza de las Ciencias, 15 (1), 43-52.

Furió, C., Solbes, J. y Carrascosa, J. (2006). Las ideas alternativas sobre conceptos científicos: tres décadas de investigación. Resultados y perspectivas. Alambique- Didáctica de las Ciencias Experimentales, 48, 64-78.

Garduño, L., López, A. y Mora, C. (2013). Evaluación del aprendizaje conceptual del movimiento de caída libre. Latin-American Journal of Physics Education, 7(2), 275-283.

George, R. (2006). A Cross domain Analysis of Change in Students' Attitudes toward Science and Attitudes about the Utility of Science. International Journal of Science Education, 28(6), 571-589. DOI: 10.1080/09500690500338755.

Gil, S. y Di Laccio, J.L. (2017). Smartphone una herramienta de laboratorio y aprendizaje: laboratorios de bajo costo para el aprendizaje de las ciencias. Latin-American Journal of Physics Education, 11, 1305-1913.

Gil, D. y Solbes, J. (1993). The introduction of modern physics: overcoming a deformed vision of science. International Journal of Science Education, 15(3), 255-260. DOI: 10.1080/0950069930150303.

González, M.A. y González, M.A. (2016). Smartphones as experimental tools to measure acoustical and mechanical properties of vibrating rods. European Journal of Physics, 37, 045701. DOI: 10.1088/0143-0807/37/4/045701.

González, M.A., González, M.A., Martín, M.E., Llamas, C., Martínez, O., Vegas, J., Herguedas, M. y Hernández, C. (2015). Teaching and Learning Physics with Smartphones. Journal of Cases on Information Technology, 17(1), 31-50. DOI: 10.4018/JCIT.2015010103.

Halloun, I.A. y Hestenes, D. (1985). Common sense concepts about motion. American Journal of Physics, 53, 465-467. DOI: 10.1119/1.14031.

Hasni, A. y Potvin, P. (2015). Student's Interest in Science and Technology and its Relationships with Teaching Methods, Family Context and Self-Efficacy. International Journal of Environmental and Science Education, 10(3), 337-366. DOI: 10.12973/ijese.2015.249a.

Hazelkorn, E., Ryan, C., Beernaert, Y., Constantinou, C.P., Deca, L., Grangeat, M., Karikorpi, M., Lazoudis, A., Pintó Casulleras, R. y Welzel-Breuer, M. (2015). Science education for responsible citizenship. Report to the European Commission of the expert group on science education. Recuperado de http://ec.europa.eu/research/swafs/pdf/pub_science_education/KINA-26-893-EN-N.pdf [19 de junio de 2019].

Hestenes, D., Wells, M. y Swackhamer, G. (1992). Force concept inventory. The Physics Teacher, 30, 141-166. DOI: 10.1119/1.2343497.

Hochberg, K., Gröber, S., Kuhn, J. y Müller, A. (2014). The spinning disc: studying radial acceleration and its damping process with smartphone acceleration sensors. Physics Education, 49(2), 137-140. DOI: 10.1088/0031-9120/49/2/137.

Jiménez, E. y Guirao, J.A. (2007). La conceptuación de la posición por los alumnos de 11 a 16 años. Propuesta de dominio de instrucción. Enseñanza de las ciencias, 25(1), 45-58.

Kuhn, J. y Müller, A. (2015). iMobilePhysics: possibilities and limits of using smartphone and tablet-PC as experimental tools. MPTL 2015, Invited Symposium iMP, contribution A41. 
Kuhn, J. y Vogt, P. (2012). Analyzing spring pendulum phenomena with a smart-phone acceleration sensor. The Physics Teacher, 50, 504-505. DOI: 10.1119/1.4758162.

Kuhn, J. y Vogt, P. (2013a). Smartphones as experimental tools: Different methods to determine the gravitational acceleration in classroom physics by using everyday devices. European Journal of Physics Education, 4(1), 16-27.

Kuhn, J. y Vogt, P. (2013b). Analyzing acoustic phenomena with a smartphone microphone. The Physics Teacher, 51, 118-119. DOI: 10.1119/1.4775539.

Kuhn, J., Vogt, P. y Hirth, M. (2014). Analyzing the acoustic beat with mobile devices. The Physics Teacher, 52, 248. DOI: 10.1119/1.4868948.

Lozano, O.R. y Solbes, J. (2014). 85 experimentos de física cotidiana. Barcelona: Graó.

Lukke Sweet, D. (2017). nonpar: A Collection of Nonparametric Hypothesis Tests. R package version 1.0.1. https://CRAN.R-project.org/package=nonpar

Monteiro, M., Stari, C., Cabeza, C. y Martí, A.C. (2015). The Atwood machine revisited using smartphones. The Physics Teacher, 53, 373. DOI: 10.1119/1.4928357.

Monteiro, M., Stari, C., Cabeza, C. y Martí, A.C. (2017). The Polarization of Light and Malus' Law Using Smartphones. The Physics Teacher, 55, 264-266. DOI: 10.1119/1.4981030.

Murphy, C. y Beggs, J. (2006). Children perceptions of school science. School Science Review, 84(308), 109-116.

Organisation for Economic Co-operation and Development (2006). Evolution of Student Interest in Science and Technology Studies Policy Report. Recuperado de http://www.oecd.org/science/sci-tech/36645825.pdf [19 de junio de 2019].

Osborne, J. y Dillon, J. (2008). Science education in Europe: critical reflections. Report to the Nuffield Foundation. London.

Osborne, J., Driver, R. y Simon, S. (1998). Attitudes to science: Issues and concerns. School Science Review, 79, 27-33.

Ostermann, F. y Moreira, M.A. (2000). Física contemporánea en la escuela secundaria: una experiencia en el aula involucrando la formación de profesores. Enseñanza de las ciencias, 18(3), $391-405$.

Parolin, S.O. y Pezzi, G. (2013). Smartphone-aided measurements of the speed of sound in different gaseous mixtures. The Physics Teacher, 51(8), 508-509. DOI: 10.1119/1.4824957.

Parolin, S.O. y Pezzi, G. (2015). Kundt's tube experiment using smartphones. Physics Education, 50(4), 508-509. DOI: 10.1088/0031-9120/50/4/443.

Pell, T. y Jarvis, T. (2001). Developing attitude to science scales for use with children of ages from five to eleven years. International Journal of Science Education, 23(8), 847- 862. DOI: 10.1080/09500690010016111.

Pitburn, M.D. y Baker, D.R. (1993). If I were the teacher...qualitative study of attitude towards science. Science Education, 77, 393-406. DOI: 10.1002/sce.3730770404.

$\mathrm{R}$ Core Team (2017). R: A language and environment for statistical computing. R Foundation for Statistical Computing, Vienna, Austria. URL https://www.R-project.org/.

Robles, A., Solbes, J., Cantó, J.R. y Lozano, O.R. (2015). Actitudes de los estudiantes hacia la ciencia escolar en el primer ciclo de la Enseñanza Secundaria Obligatoria. Revista Electrónica de Enseñanza de las Ciencias, 14(3), 361-376.

Rocard, M., Csermely, P., Jorde, D., Lenzen, D., Walberg-Henriksson, H. y Hemmo, V. (2007). Science education now: A renewed pedagogy for the future of Europe. Report to the European Commission of the expert group on science education. Recuperado de http://ec.europa.eu/research/science-society/document_library/pdf_06/report-rocard-onscience-education_en.pdf [19 de junio de 2019].

Rosenthal, R. (1994). Parametric measures of effect size. En H. Cooper y L.V. Hedges (Eds.), The handbook of research synthesis (pp. 231-244). Nueva York: Russell Sage Foundation. 
Salinas, I., Giménez, M.H., Monsoriu, J.A. y Castro-Palacio, J.C. (2018). Characterization of linear light sources with the smartphone's ambient light sensor. The Physics Teacher, 56, 562-563. DOI: 10.1119/1.5064575.

Sans, J.A., Gea-Pinal, J., Giménez, M.H., Esteve, A.R., Solbes, J. y Monsoriu, J.A. (2017). Determining the efficiency of optical sources using a smartphone's ambient light sensor. European Journal of Physics, 38(2), 025301. DOI: 10.1088/1361-6404/aa51a9.

Santos, J.V. y Gras-Martí, A. (2003). Conocimientos de Física de alumnos universitarios. Influencia de las reformas educativas. Revista Electrónica de Enseñanza de las Ciencias, 2(2), 126-135.

Sebastià, J.M. (1984). Fuerza y Movimiento, la interpretación de los estudiantes. Enseñanza de las Ciencias, 2(3), 161-169.

Simpson, R.D. y Oliver, J.E. (1990). A Summary of Major Influences on Attitude Toward and Achievement in Science Among Adolescent Students. Science Education, 74(1), 1-18. DOI: 10.1002/sce.3730740102.

Sitar, D. (2012). Imaging Emission Spectra with Handheld and Cellphone Cameras. The Physics Teacher, 50(9), 524-525. DOI: 10.1119/1.4767480.

Solbes, J. y Vilches, A. (2004). Papel de las relaciones entre ciencia, tecnología, sociedad y ambiente en la formación ciudadana. Enseñanza de las Ciencias, 22(3), 337-348.

Solbes, J., Montserrat, R. y Furió, C. (2007). El desinterés del alumnado hacia el aprendizaje de la ciencia: implicaciones en su enseñanza. Didáctica de las Ciencias Experimentales y Sociales, 21, 91-117.

Thoms, L.J., Colicchia, G. y Girwidz, R. (2013). Color reproduction with a smartphone. The Physics Teacher, 51(7), 440-441. DOI: 10.1119/1.4820866.

Vázquez, A. y Manassero, M.A. (2008). El declive de las actitudes hacia la ciencia de los estudiantes: un indicador inquietante para la educación científica. Revista Eureka sobre Enseñanza y Divulgación de las Ciencias, 5(3), 274-292.

Viennot, L. (1979). Le raisonnement spontané en dynamique élémentaire. Paris: Hermann.

Vieyra, R.E. y Vieyra, C. (2014). Analyzing Forces on Amusement Park Rides with Mobile Devices. The Physics Teacher, 52, 149-151. DOI: 10.1119/1.4865516.

Vogt, P. y Kuhn, J. (2012a). Analyzing free fall with a smartphone acceleration sensor. The Physics Teacher, 50, 182-183. DOI: 10.1119/1.3685123.

Vogt, P. y Kuhn, J. (2012b). Analyzing simple pendulum phenomena with a smartphone acceleration sensor. The Physics Teacher, 50, 439-440. DOI: 10.1119/1.4752056.

Vogt, P. y Kuhn, J. (2014). Analyzing collision processes with the smartphone acceleration sensor. The Physics Teacher, 52, 118-119. DOI: 10.1119/1.4862122.

\section{CÓMO CITAR ESTE ARTÍCULO}

Esteve, A. R., Benavent, A. y Solbes, J. (2019). Smartphones y caída libre: Diseño y evaluación de una experiencia práctica. Didáctica de las ciencias experimentales y sociales, 37, 165-178. DOI: 10.7203/DCES.37.15441 
